\title{
Influence of bulk dielectric polarization upon partial discharge transients: Effect of void geometry and orientation
}

\author{
McAllister, lain Wilson; Crichton, G.C.
}

Published in:

IEEE Transactions on Dielectrics and Electrical Insulation

Link to article, DOI:

10.1109/TDEI.2005.1430394

Publication date:

2005

Document Version

Publisher's PDF, also known as Version of record

Link back to DTU Orbit

Citation (APA):

McAllister, I. W., \& Crichton, G. C. (2005). Influence of bulk dielectric polarization upon partial discharge transients: Effect of void geometry and orientation. IEEE Transactions on Dielectrics and Electrical Insulation, 12(2), 240-247. https://doi.org/10.1109/TDEl.2005.1430394

\section{General rights}

Copyright and moral rights for the publications made accessible in the public portal are retained by the authors and/or other copyright owners and it is a condition of accessing publications that users recognise and abide by the legal requirements associated with these rights.

- Users may download and print one copy of any publication from the public portal for the purpose of private study or research.

- You may not further distribute the material or use it for any profit-making activity or commercial gain

- You may freely distribute the URL identifying the publication in the public portal 


\title{
Influence of Bulk Dielectric Polarization upon Partial Discharge Transients: Effect of Void Geometry and Orientation
}

\author{
I. W. McAllister and G. C. Crichton \\ Department of Electric Power Engineering \\ Technical University of Denmark \\ DK-2800 Lyngby, Denmark
}

\begin{abstract}
The induced charge arising from a partial discharge consists of 2 components. One is associated with the actual space charge in the void. The other is related to changes in the polarization of the bulk dielectric. These changes are a direct consequence of the field produced by the space charge. The influence of the void geometry upon the polarization component of the induced charge is examined for a heterogeneous bulk dielectric system. It is demonstrated that, depending on the ratio of the dielectric permittivities and within which dielectric the void is located, the relative magnitude of this component may increase or decrease. The magnitude of this effect is also strongly dependent upon the prolateness/oblateness of the void geometry, and on the orientation of the void with respect to the detecting electrode.

Index Terms - Partial discharge transients, spheroidal voids, heterogenous bulk dielectric, dielectric polarization.
\end{abstract}

\section{INTRODUCTION}

A partial discharge in a gaseous void produces a change in the polarization $(\delta \vec{P})$ of the bulk dielectric. This change is reflected in the resultant magnitude of the charge induced upon the detecting electrode [1-3].

The influence of the dielectric-system geometry upon $\delta \vec{P}$ has been examined previously in [3]. In the present paper, the influence of the void geometry upon $\delta \vec{P}$ is examined for a two-layer, bulk dielectric system. It is again shown that the component of the induced charge due to $\delta \vec{P}$ may increase or decrease depending upon the ratio of the dielectric permittivities and within which dielectric the void is located. Apart from the influence of the permittivity ratio, the magnitude of the effect is also strongly dependent upon both the prolateness/oblateness of the void geometry, and on the void orientation with reference to the detecting electrode. Through the polarization component of the induced charge, this behaviour is directly reflected in the Poissonian induced charge [4], and consequently in the recorded partial discharge transient.

\section{MATHEMATICAL PRELIMINARIES}

The theory behind the influence of changes in polarization is fully described in [3]. However to enable the ar-

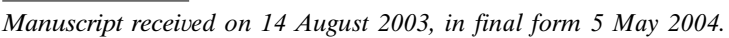

guments presented in this paper to be readily followed, a brief description of the essential theory will be given below.

\subsection{POLARIZATION COMPONENT OF THE POISSONIAN INDUCED CHARGE}

The Poissonian induced charge is that component of the induced charge which is rigidly linked to the space charge source [4]. Mathematically, the final value of the Poissonian induced charge $q$ due to a partial discharge may be resolved into two components [2], viz.

$$
q=q_{\mu}+q_{P}
$$

where $q_{\mu}$ is the induced charge directly associated with the space charge in the void, and $q_{P}$ represents the induced charge related to the change in dielectric polarization $(\delta \vec{P})$ due to the presence of this space charge [2].

With reference to the detecting electrode and the induced charge, the effect of the void wall charges arising from the partial discharge can be equated to the effect of an electric dipole of moment $\vec{\mu}$ located within the void [1]. The total Poissonian induced charge arising from a dipole is given by

$$
q=-\vec{\mu} \cdot \vec{\nabla} \lambda
$$

where $\lambda$ is the proportionality factor between the charge in the void and the induced charge on the detecting elec- 
trode. The $\lambda$ function, which is a solution of the general Laplace equation [2], takes account of the dielectric polarization inherently.

With another proportionality factor, $\varphi$, it is possible to determine the component of the Poissonian induced charge related to the void space charge alone, viz. we have

$$
q_{\mu}=-\vec{\mu} \cdot \vec{\nabla} \varphi
$$

The $\varphi$ function, which is a solution of the reduced Laplace equation [2], could also be used to account for the dielectric polarization explicitly.

However, from (1), (2) and (3), the polarization component $q_{P}$ of the Poissonian induced charge may be readily expressed as

$$
q_{P}=-\vec{\mu} \cdot(\vec{\nabla} \lambda-\vec{\nabla} \varphi)
$$

\subsection{THE $\lambda_{0}$ FUNCTION}

If the dimensions of the void are such that $\vec{\nabla} \lambda$ can be assumed constant within the void, then we may introduce another function, $\lambda_{0}$. This function, which is derived for the same boundary conditions but without the void, represents the unperturbed $\lambda$ function. As both $\lambda$ and $\lambda_{0}$ are solutions of Laplace's equation, then by mathematical analogy with electrostatic fields, the relation between the $\lambda$ and the $\lambda_{0}$ functions is given by

$$
\vec{\nabla} \lambda=h \vec{\nabla} \lambda_{0}
$$

For the type of void under consideration, the parameter $h$ is a scalar which depends on the void geometry and the relative permittivity of the associated bulk medium. Following the introduction of $\lambda_{0}$, the polarization component of the Poissonian induced charge may be expressed as

$$
q_{P}=-\vec{\mu} \cdot\left(h \vec{\nabla} \lambda_{0}-\vec{\nabla} \varphi\right)
$$

\subsubsection{HOMOGENEOUS BULK DIELECTRIC SYSTEM}

For a homogeneous bulk dielectric system, $\lambda_{0}$ becomes a solution of the reduced Laplace equation. In such situations $\lambda_{0}$ and $\varphi$ are synonomous and hence (6) reduces to

$$
q_{P}=-(h-1) \vec{\mu} \cdot \vec{\nabla} \lambda_{0}
$$

Combining equations (2), (5) and (7), we obtain the relationship

$$
\frac{q_{P}}{q}=1-\frac{1}{h}
$$

For the voids under consideration, $h>1$. This implies that for a homogeneous bulk dielectric, $q_{P} / q$ is always positive, i.e. $q_{P} / q>0$. The effect of void geometry upon

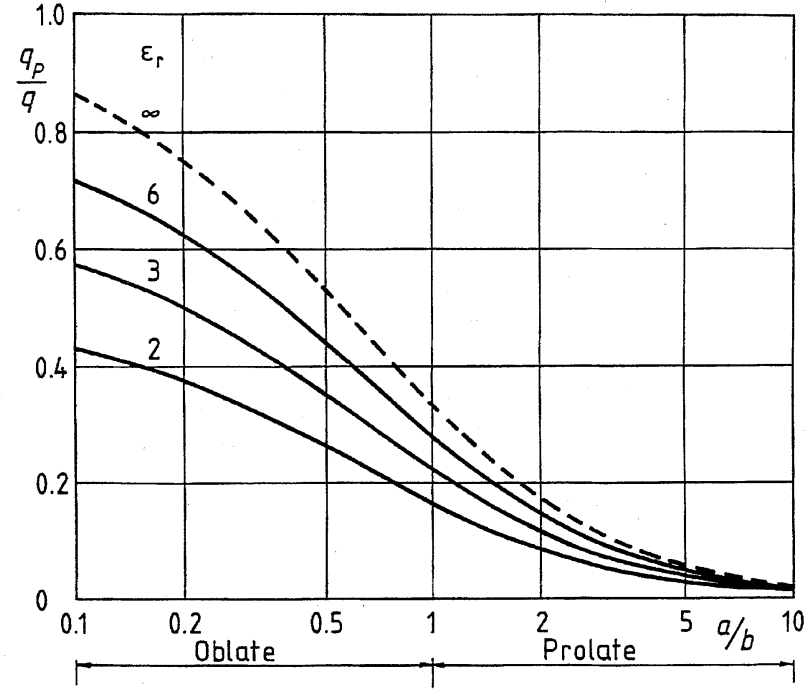

(a)

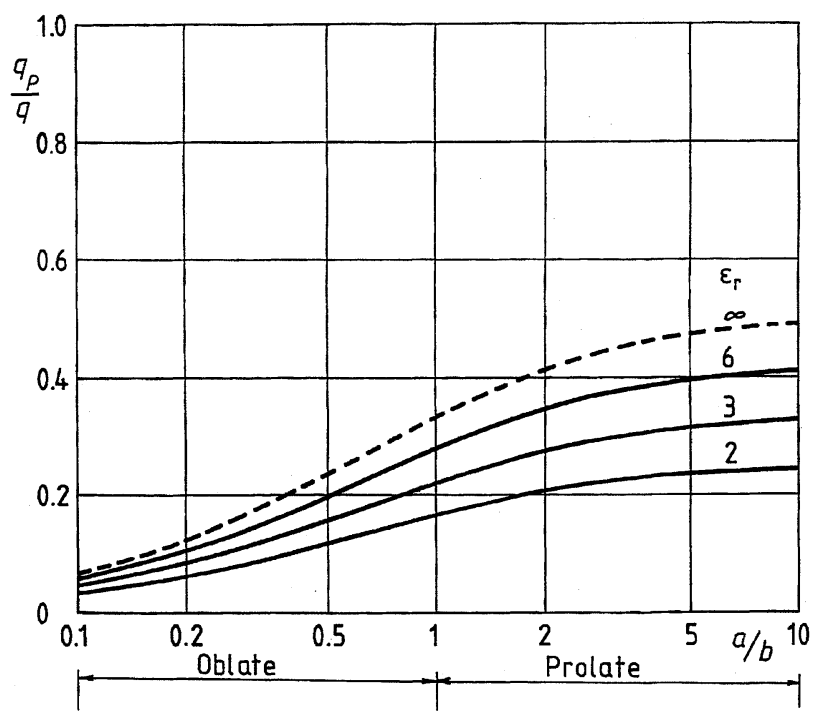

(b)

Figure 1. Variation of $q_{P} / q$ for spheroidal voids in a homogeneous bulk dielectric system [2]. a, parallel orientation of the void; b, transverse orientation of the void.

the behaviour of $q_{P}$ for a homogeneous system has been reported in [2], and for reference the variations are shown in Figure 1. These $q_{P} / q$ variations are associated with spheroidal voids, for which $a$ and $b$ represent the semiaxes of the voids, with $a$ being the axis of rotation.

\subsubsection{HETEROGENEOUS BULK DIELECTRIC SYSTEM}

To examine the situation in which $\lambda_{0}$ is a solution of the general Laplace equation, we consider initially a general heterogeneous dielectric system. However, as indicated by (6), the $q_{P}$ expression involves three independent vectors, and thus the general behaviour of $q_{P}$ is difficult 


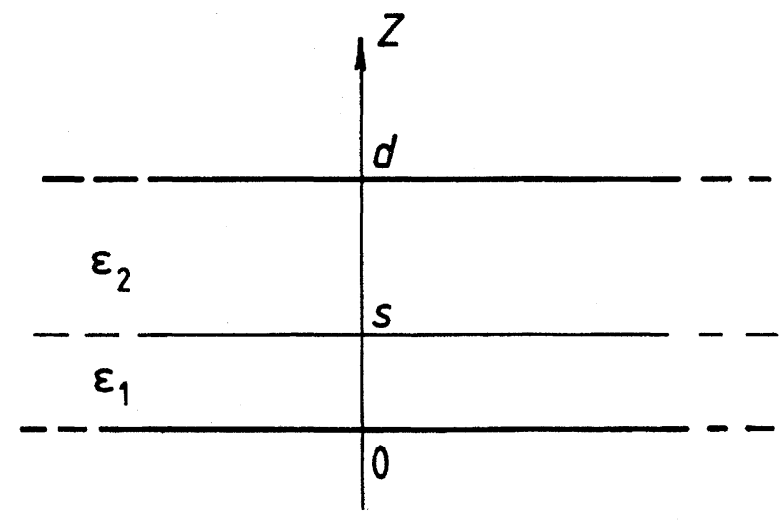

Figure 2. Two-layer planar dielectric system.

to elucidate. Hence in this study, we will limit ourselves to situations for which the three vectors are constrained to be parallel.

A simple geometry which fulfils this requirement is shown in Figure 2; namely a planar geometry which consists of a two layer dielectric system. If in rectangular coordinates, the electrodes are represented by $z=0$ and $z=d$, then the dielectric interface is taken as $z=s$, with $s$ $<d$. The permittivity of the upper dielectric is $\epsilon_{2}$ for which $s \leq z \leq d$, while that of the lower is $\epsilon_{1}$, for which $0 \leq z \leq s$. If the lower electrode is used as the detecting electrode, then the boundary conditions for the $\lambda_{0}$ function are $\lambda_{0}=1$ for $z=0$ and $\lambda_{0}=0$ for $z=d$. For this geometry, the derivation of the relevant $\vec{\nabla} \lambda_{0}$ and $\vec{\nabla} \varphi$ functions is straightforward, and the relevant expressions may be found in [3].

For a heterogeneous system, the influence of $\delta \vec{P}$ upon partial discharge transients may be illustrated by making a comparison with the corresponding homogeneous system, see [3] for details. Such a comparison is however independent of the void geometry, and is thus not of relevance for the present study.

\section{INDUCED CHARGE COMPONENT DUE TO $\delta \vec{P}$ IN HETEROGENEOUS DIELECTRIC SYSTEMS}

\subsection{INTRODUCTION}

To ensure that the concept of $h$ and (5) are valid, it will be assumed that, in the heterogeneous bulk dielectric, the void is more than 10 times its greatest linear dimension from any interface, such that the $\vec{\nabla} \lambda$ distribution within the void remains effectively uniform; i.e. the existence of an interface does not perturb $\vec{\nabla} \lambda$ in the void.

With respect to the component of the induced charge related to $\delta \vec{P}$, we obtain upon combining (2), (5) and (6) the following general relation between $q_{P}$ and $q$, viz.

$$
\frac{q_{P n}}{q_{n}}=\frac{-\vec{\mu} \bullet\left(h_{n} \vec{\nabla} \lambda_{0 n}-\vec{\nabla} \varphi\right)}{-\vec{\mu} \bullet h_{n} \vec{\nabla} \lambda_{0 n}}
$$

where $q_{n}$ is the Poissonian induced charge of the heterogeneous system, and $n=1,2$ depending in which dielectric the void is located.

On account of the planar geometry, the resulting dipole moment will possess only a $z$ component. This may be directed either away from, or towards the coordinate origin. However, without loss of generality, we select the positive $z$ direction; viz.

$$
\vec{\mu}=\mu \vec{e}_{z}
$$

where $\vec{e}_{z}$ is a unit vector in the positive $z$ direction (see Figure 2). Performing the vector operations enables (9) to be reduced to

$$
\frac{q_{P n}}{q_{n}}=1-\frac{d \varphi / d z}{h_{n} d \lambda_{0 n} / d z}
$$

Provided the use of $h_{n}$ in (5) remains valid, then the presence of $h$ in (11) implies that $q_{P n} / q_{n}$ is dependent upon the void geometry.

From (11) it is also clear that the polarity of $q_{P n} / q_{n}$ is dependent upon whether

$$
\frac{d \varphi / d z}{h_{n} d \lambda_{0 n} / d z}<1
$$

or

$$
\frac{d \varphi / d z}{h_{n} d \lambda_{0 n} / d z}>1
$$

Equations (12a) and (12b) show that the polarization component may reverse polarity. The polarity of the Poissonian induced charge does not, however, exhibit such behaviour [3].

In [3] it is shown that, for a void in medium 1 , we have

$$
\frac{q_{P 1}}{q_{1}}=1-\frac{\epsilon_{1}(d-s)+\epsilon_{2} s}{\epsilon_{2} h_{1} d}
$$

while, for a void in medium 2 , the corresponding relation is

$$
\frac{q_{P 2}}{q_{2}}=1-\frac{\epsilon_{1}(d-s)+\epsilon_{2} s}{\epsilon_{1} h_{2} d}
$$

Apart from permittivity and layer dimensions, both (13) and (14) contain $h$. This confirms that $q_{P n} / q_{n}$ is also dependent upon the specific void geometry. For $\epsilon_{1} \equiv \epsilon_{2}$, and subsequently $h_{1} \equiv h_{2}$, both (13) and (14) reduce to (8).

\subsection{INFLUENCE OF VOID GEOMETRY}

The influence of the void geometry upon $q_{P n}$ will be studied with reference to spheroidal voids. For such voids, $h$ is given by

$$
h=\frac{K \epsilon_{r}}{1+(K-1) \epsilon_{r}}
$$

where $\epsilon_{r}$ is the relative permittivity of the relevant bulk dielectric and $K$ is a dimensionless parameter which arises from the analysis [5]. 
For oblate spheroidal voids we have $[5,6]$

$$
\begin{aligned}
K_{p} & =\frac{u^{3}}{\left(u^{2}+1\right)(u-\arctan u)} \\
K_{t} & =\frac{2 u^{3}}{\left(u^{2}+1\right) \arctan u-u}
\end{aligned}
$$

where the subscripts $p$ and $t$ refer to $\vec{\nabla} \lambda_{0}$ and the applied field being either parallel to, or transverse to the spheroid axis of rotation. The variable $u$ is given by

$$
u=\sqrt{(b / a)^{2}-1}
$$

where $a$ and $b$ are the semi-axes of the oblate spheroid such that $b / a>1$, i.e. $a$ is the axis of rotation.

For a prolate spheroidal void $(b / a<1)$, the associated expressions for $K$ are [5,6]

$$
\begin{gathered}
K_{p}=\frac{2 \nu^{3}}{\left(1-\nu^{2}\right)\left[\ln \left(\frac{1+\nu}{1-\nu}\right)-2 \nu\right]} \\
K_{t}=\frac{4 \nu^{3}}{\left(1-\nu^{2}\right) \ln \left(\frac{1-\nu}{1+\nu}\right)+2 \nu}
\end{gathered}
$$

with

$$
\nu=\sqrt{1-(b / a)^{2}}
$$

and $a$ is again the axis of rotation. With the knowledge of the $K$ values, (15) enables the corresponding $h$ values to be derived and thereafter inserted into (13) and (14). The variation of $h$ with $a / b$ is illustrated in [6] (Figure 3).

\section{RESULTS AND DISCUSSION \\ 4.1 INFLUENCE OF PERMITTIVITY RATIO}

The variations of $q_{P n} / q_{n}$ with $\epsilon_{2} / \epsilon_{1}$ are shown in Figures 3 and 4 for the principal orientations of the voids with respect to $\vec{\nabla} \lambda_{0}$, and several values of $s / d$. Figure 3 refers to prolate $(a / b=5 / 1)$ voids, while Figure 4 relates to oblate $(a / b=1 / 5)$ voids.

The behaviour of $q_{P n} / q_{n}$ is similar to that reported previously for a spherical void [3]: i.e. $q_{P n}$ is increased when the void is in a medium of lesser permittivity, while a decrease occurs when located in the medium of greater permittivity. Depending upon the $q_{P n} / q_{n}$ crossover value: i.e. the value associated with the corresponding homogeneous system $\left(\epsilon_{2} / \epsilon_{1}=1\right)$, see Figure 1 , this reduction in $q_{P n} / q_{n}$ can be so marked that the polarity of $q_{P n}$ is reversed. This behaviour is examined in more detail in Section 4.3.

The influence of the void orientation is evident in both Figures. This behaviour is most readily understood with reference to Figure 1 for the homogeneous bulk dielectric. For example with $\epsilon_{r}=4$, a prolate void of $a / b=$

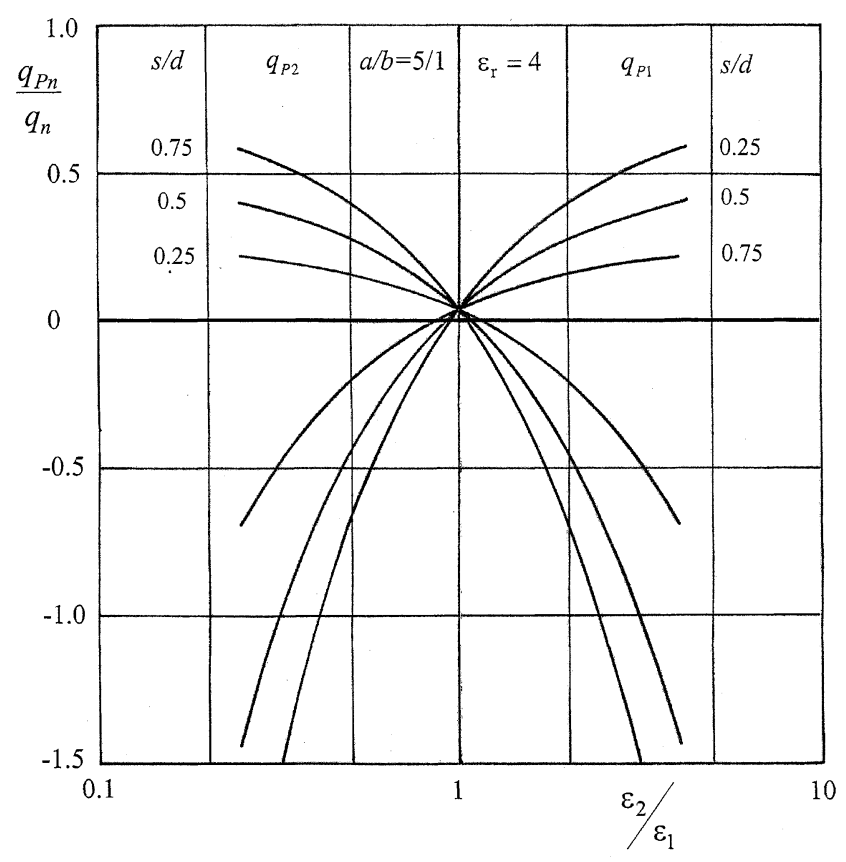

(a)

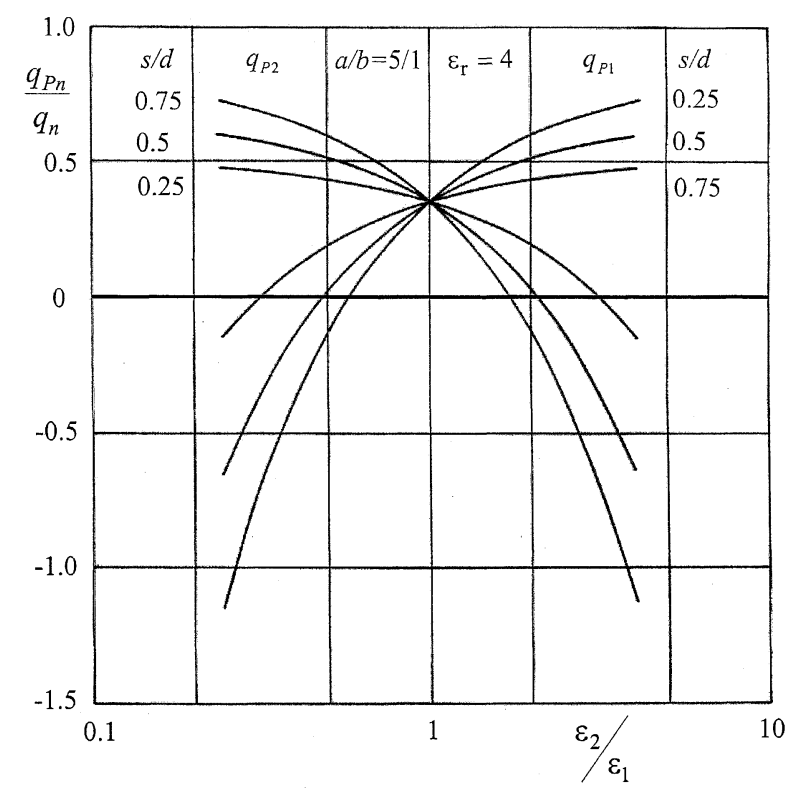

(b)

Figure 3. Variation of with $q_{P n} / q_{n}$ with $\epsilon_{2} / \epsilon_{1}$ for prolate spheroidal voids. a, parallel orientation of the void; $\mathrm{b}$, transverse orientation of the void.

$5 / 1, q_{P} / q \sim 0.04$ for the parallel orientation, while $q_{P} / q$ $\sim 0.35$ for the transverse orientation. Similarly for the oblate void of $a / b=1 / 5$, the related values of $q_{P} / q$ are $\sim 0.5$ and $\sim 0.01$, respectively.

The value of $s / d$ represents the relative thickness of medium 1, and hence the results shown in Figures 3 and 4 imply that, when the void is in the medium of smaller physical extent, the greater is the relative increase/de- 


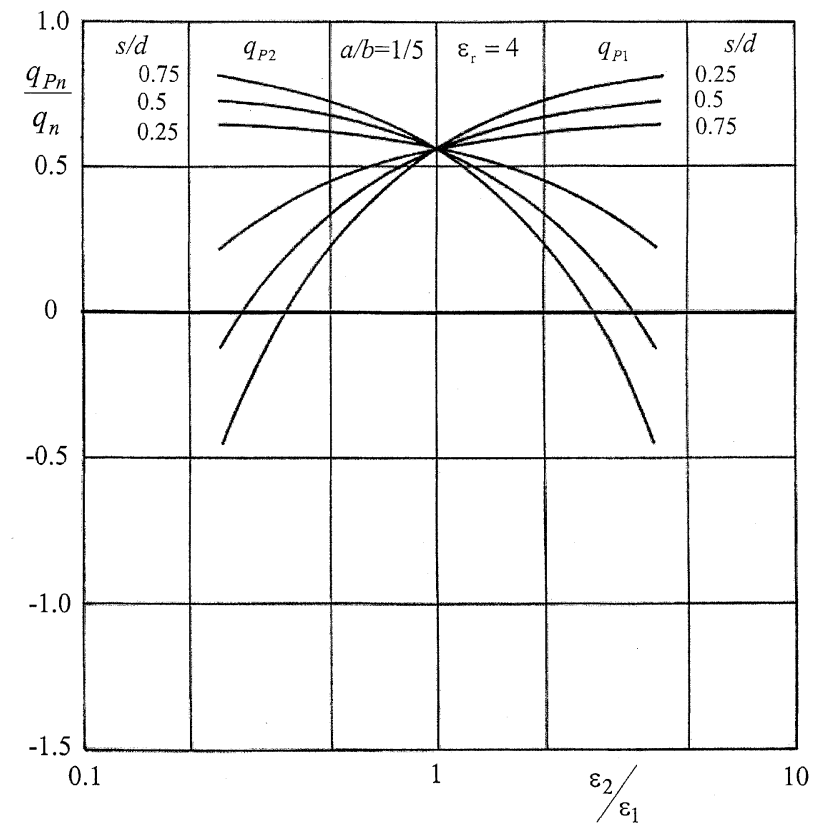

(a)

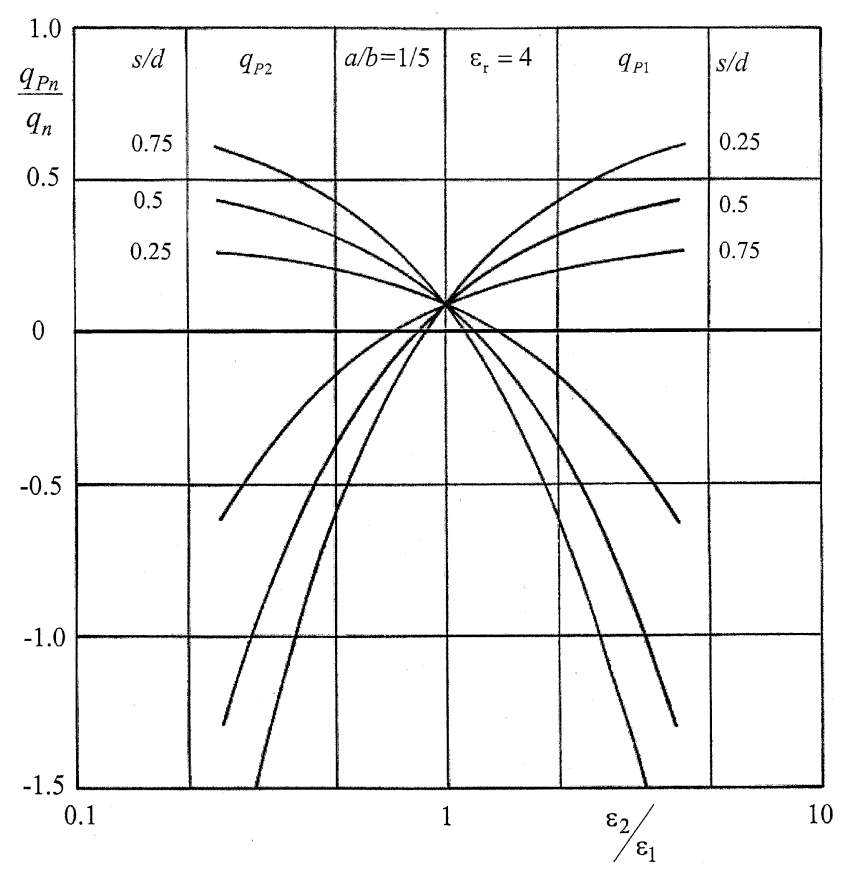

(b)

Figure 4. Variation of $q_{P n} / q_{n}$ with $\epsilon_{2} / \epsilon_{1}$ for oblate spheroidal voids. a, parallel orientation of the void; $b$, transverse orientation of the void.

crease in $q_{P n}$. Owing to the simple geometry under discussion, see [3], the behaviour of $q_{P n}$ is symmetrical with respect to $s / d$ and $(1-s / d)$.

Similar variations of $q_{P n} / q_{n}$ are displayed by other values of $\epsilon_{r}$. The actual $q_{P n} / q_{n}$ magnitudes are related to the homogeneous values shown in Figure 1, as these val- ues represent the $q_{P n} / q_{n}$ crossover values of Figures $3 \&$ 4. From Figure 1, it is evident that $\epsilon_{r}>4$ leads to increased $q_{P n} / q_{n}$ values, while for $\epsilon_{r}<4$, these values will be decreased.

\subsection{INFLUENCE OF VOID GEOMETRY}

The variation of $q_{P 1} / q_{1}$ with $a / b$ is shown in Figure 5 for the principal orientations of the voids with respect to $\vec{\nabla} \lambda_{0}$ and selected values of $\epsilon_{2} / \epsilon_{1}$. Figure 5a refers to the parallel orientation of the voids, while Figure $5 \mathrm{~b}$ relates to the transverse orientation. In discussing the results of Figure 5, it proves convenient to take the homogeneous case $\left(\epsilon_{2} / \epsilon_{1}=1\right)$ as a reference situation.

With respect to the parallel orientation, it is evident from Figure 5a that, for $\epsilon_{2} / \epsilon_{1}>1, q_{P 1}$ is increased, with the prolate void $(a / b>1)$ exhibiting the greater relative effect. For $\epsilon_{2} / \epsilon_{1}<1$, there is a reduction in $q_{P 1}$. This can be so marked that the polarity of $q_{P 1}$ is reversed. Here, the prolate void displays the greater reductions, both in relative and absolute terms.

For the transverse orientation, Figure $5 \mathrm{~b}$ indicates that, for $\epsilon_{2} / \epsilon_{1}>1, q_{P 1}$ is also increased. However now it is the oblate void $(a / b<1)$ which exhibits the greater relative effect. For $\epsilon_{2} / \epsilon_{1}<1$, there is again a reduction in $q_{P 1}$. As previously, this can be of such a degree that the polarity of $q_{P 1}$ is reversed. In this condition, the oblate void exhibits the greater reductions, both in relative and absolute terms.

A comparison of Figure 5a with Figure 5b indicates that these results are essentially the mirror image of each other. Moreover, with reference to Figure 5, if we interchange the $\epsilon$ subscripts 2 and 1, and consider the void to be in medium 2, then this Figure would display the variations of $q_{P 2} / q_{2}$ as a function of $a / b$.

For other $\epsilon_{r}$ values, similar variations of $q_{P 1} / q_{1}$ with $a / b$ are obtained. The actual differences can be discussed with reference to the homogeneous variations shown in Figure 1. For $\epsilon_{r}>4$, the value of $q_{P 1} / q_{1}$ is increased, while for $\epsilon_{r}<4$, there is a reduction. For the parallel orientation, the greatest increases/decreases relate to oblate voids, whereas prolate voids exhibit this feature for the transverse orientation. Such behaviour is in keeping with that shown in Figures 1a and 1b, respectively.

Although different values of $s / d$ lead to similar variations of $q_{P 1} / q_{1}$ with $a / b$ as shown in Figure 5 , there is a considerable difference in the magnitudes of $q_{P 1} / q_{1}$. This behaviour can be understood through Figures 3 and 4 . Therefore, depending on whether $\epsilon_{2} / \epsilon_{1}>1$ or $\epsilon_{2} / \epsilon_{1}<1$ it is evident that the smaller $s / d$ is, the greater are the increases/decreases in $q_{P 1} / q_{1}$. The greater $s / d$ is, the smaller are these effects. A similar behaviour is exhibited by $q_{P 2} / q_{2}$ when reference is made to $\epsilon_{1} / \epsilon_{2}$ and $(1-s / d)$ instead of $\epsilon_{2} / \epsilon_{1}$ and $s / d$. 


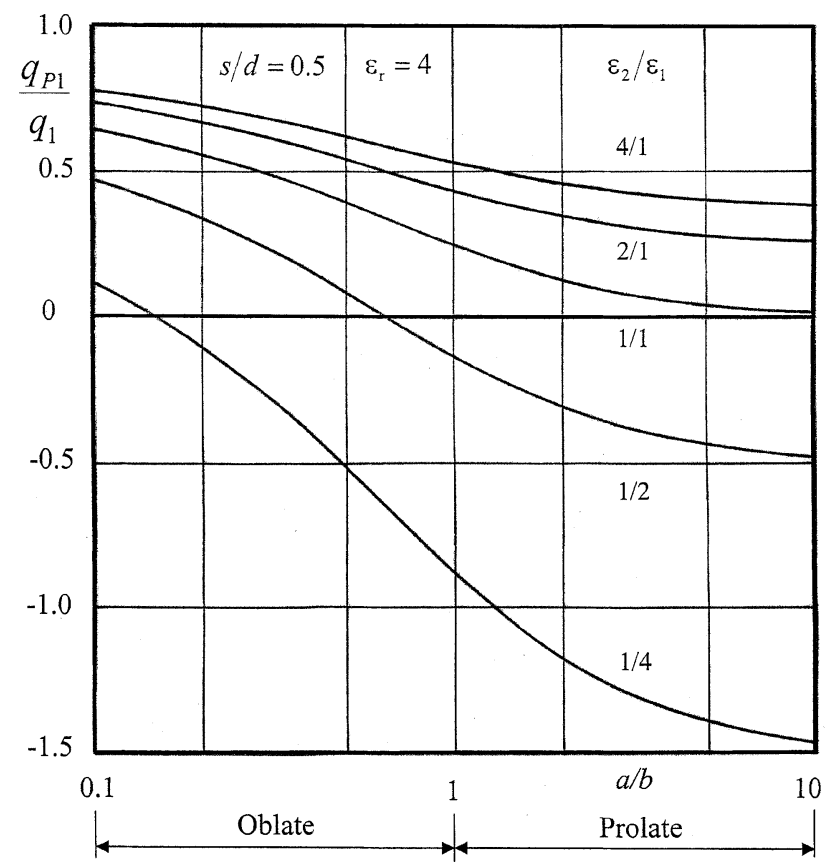

(a)

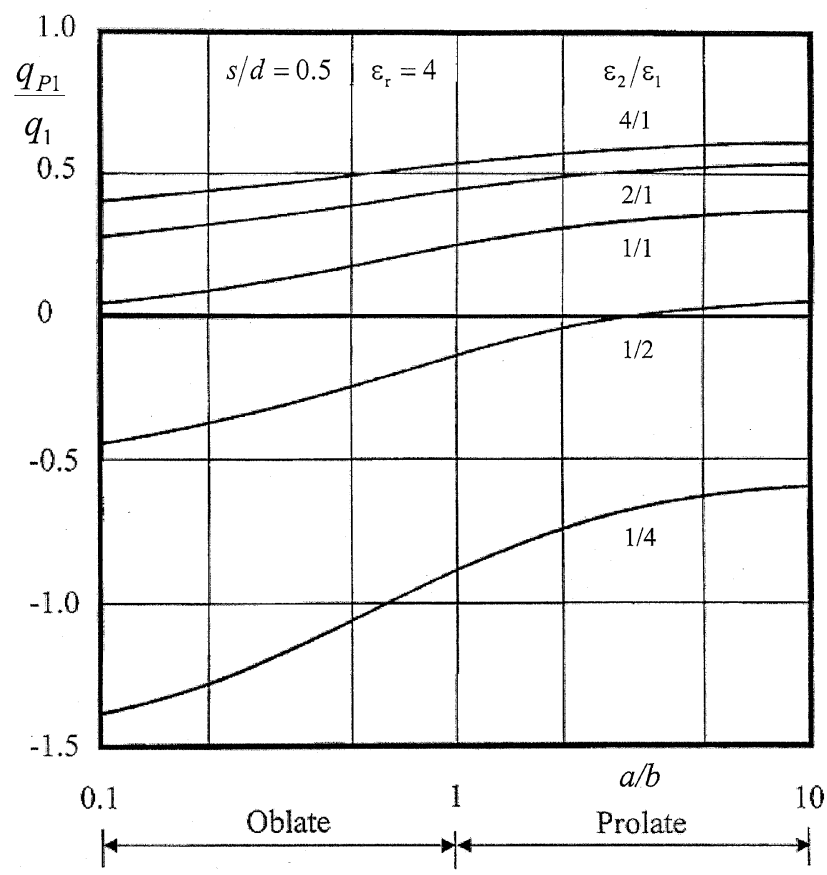

(b)

Figure 5. Variation of $q_{P 1} / q_{1}$ with $a / b$ for selected $\epsilon_{2} / \epsilon_{1}$ values. $a$, parallel orientation of the void; $b$, transverse orientation of the void.

\subsection{POLARITY REVERSAL OF $q_{P n}$}

From Figure 5, it is evident that for a range of values of the permittivity ratio $\epsilon_{2} / \epsilon_{1}$, the value of $q_{\mathrm{P} 1} / q_{1}$ may be zero. To obtain the relevant ratio values, we equate the right hand side of equations (13) and (14) to zero. Conse- quently, for $q_{P 1} / q_{1}=0$, we obtain from (13) the relationship

$$
\left(\epsilon_{2} / \epsilon_{1}\right)_{c}=\frac{d-s}{d h_{1}-s}
$$

while, for $q_{P 2} / q_{2}=0$, we have from equation (14)

$$
\left(\epsilon_{1} / \epsilon_{2}\right)_{c}=\frac{s}{d\left(h_{2}-1\right)+s}
$$

The subscript ' $c$ ' in equations (22) and (23) refers to the fact that the permittivity ratio has a critical value. As the polarity reversal of $q_{P n}$ occurs when the void is in the dielectric of greater permittivity, this implies that the values of the ratios in equations (22) and (23) are $<1$, and that the permittivity in the denominator of the critical ratio represents the medium containing the void.

The variations of the above permittivity ratios with $a / b$ for different $\epsilon_{r}$ values are shown in Figure 6. From this

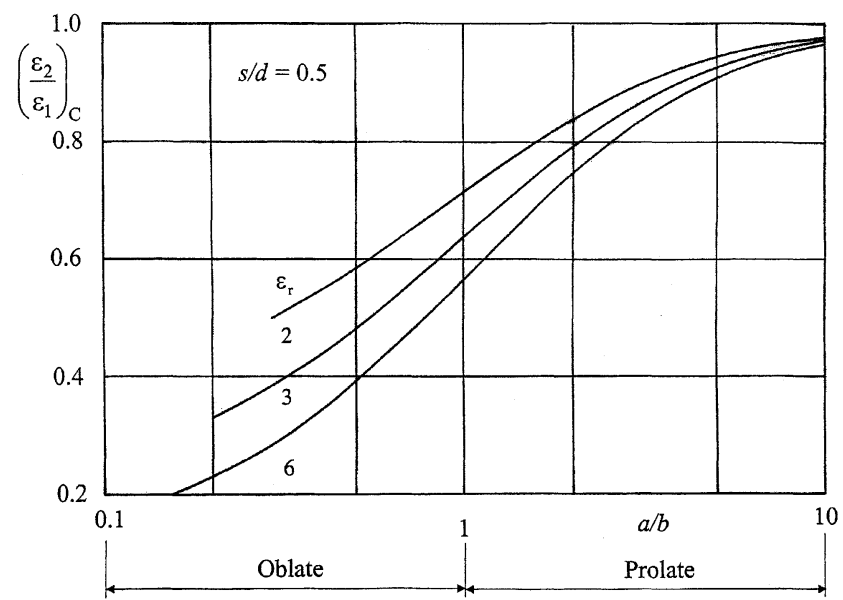

(a)

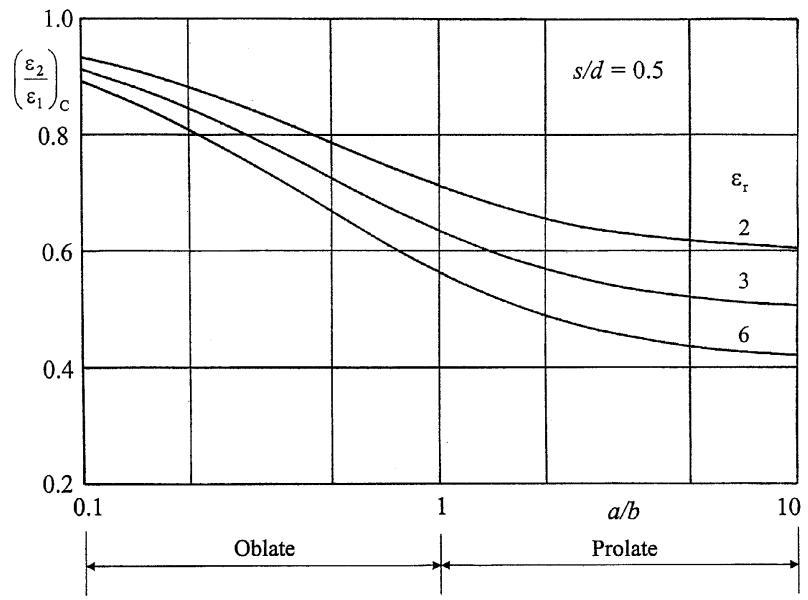

(b)

Figure 6. Variation of the 'zero' permittivity ratios with $a / b$ for selected $\epsilon_{r}$ values. a, parallel orientation of the void; $b$, transverse orientation of the void. 


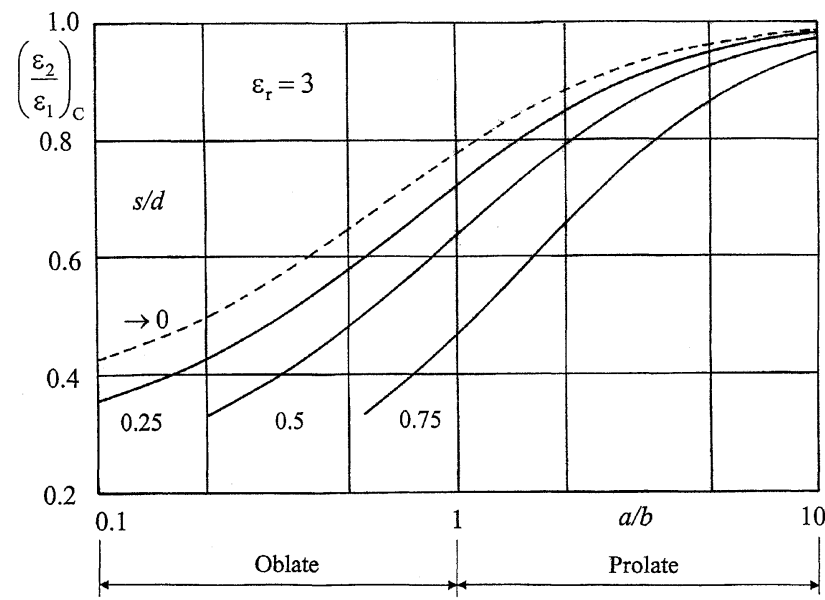

(a)

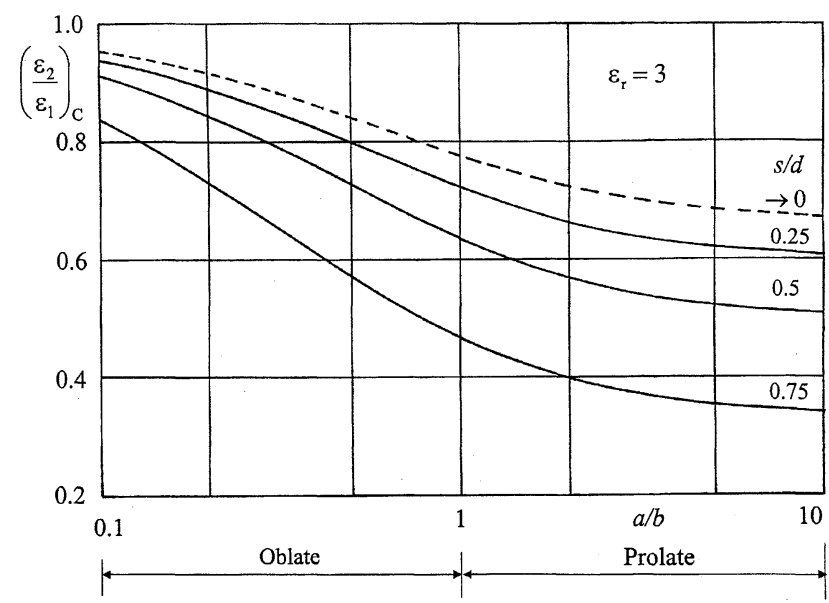

(b)

Figure 7. Variation of the 'zero' permittivity ratios with $a / b$ for selected $s / d$ values. a, parallel orientation of the void; $b$, transverse orientation of the void

Figure, the influence of void geometry and orientation are evident. For the parallel orientation, a $\left(\epsilon_{2} / \epsilon_{1}\right)_{c}$ value of $\sim 0.9$ is sufficient to provide a polarity reversal in $q_{P 1} / q_{1}$ with prolate voids. This condition can be observed in Figure $3 \mathrm{a}$ for $a / b=5 / 1$. For oblate voids, the values of $\left(\epsilon_{2} / \epsilon_{1}\right)_{c}$ are smaller, $<0.7$. This situation is related to the greater value of $q_{P} / q$ in the homogeneous system, e.g. see Figure $3 b$ for $a / b=1 / 5$.

For the transverse orientation, the range of $\left(\epsilon_{2} / \epsilon_{1}\right)_{c}$ is less, and now the sensitivity of the voids to a polarity change is reversed: i.e. the oblate void is associated with the greater values of $\left(\epsilon_{2} / \epsilon_{1}\right)_{c}$.

The influence of $s / d$ upon the permittivity ratios for $\epsilon_{r}$ $=3$ is illustrated in Figure 7. Also included is the limiting value of the ratio as $s / d \rightarrow 0$, see [3]. This Figure indicates that the relative dimensions of the bulk dielectrics are a significant parameter in determining the value of $\left(\epsilon_{2} / \epsilon_{1}\right)_{c}$. Owing to the symmetry of the system considered, the $\left(\epsilon_{2} / \epsilon_{1}\right)_{\mathrm{c}}$ variations illustrated in Figures 6 and 7 are in effect also these of $\left(\epsilon_{2} / \epsilon_{1}\right)_{c}$ when $s / d$ is replaced by (1$s / d)$.

\section{DISCUSSION}

\subsection{GENERAL REMARKS}

Mathematically, the Poissonian induced charge from a PD consists of two components [2]. One component is related directly to the space charge created by the PD in the void. The other is associated with the change in the polarization of the bulk dielectric produced by the electric field of the PD space charge. Through the use of the $\lambda$ and $\varphi$ functions, quantitative expressions for the polarization component of the induced charge can be developed, viz. (4) or (6).

However, as each of these expressions contains three independent vectors, the general behaviour of the polarization component for heterogeneous bulk dielectric systems, is not readily elucidated. Nevertheless to gain a measure of insight, the present study has addressed the condition in which the three vectors are parallel. For a heterogeneous bulk dielectric system, this requirement can be met by a planar electrode system.

With respect to the influence of a heterogeneous dielectric system upon the polarization component, the basic results are identical with those reported previously [3]: i.e. the relative magnitude of the polarization component of the induced charge may increase or decrease depending on the ratio of the bulk dielectric permittivities, and within which dielectric the void is located. When located in the dielectric of greater permittivity, the reduction can be so great that the polarity of the polarization component is reversed.

The polarization component is also influenced by the relative physical dimensions of the dielectric containing the void; viz. the smaller this dielectric is in extent, the larger is the change in magnitude. This sensitivity is associated with the fact that the basis of $q_{P}$ is a volume integral involving the changes in the bulk dielectric polarization produced by the partial discharge, see [2].

\subsection{INFLUENCE OF VOID GEOMETRY UPON THE POLARIZATION COMPONENT}

As indicated by equation (9), the ratio of the polarization component to the Poissonian induced charge $\left(q_{P n} / q_{n}\right)$ is dependent on the void geometry, and thus this expression was used to study the influence of the void geometry. The results indicate that the magnitude and polarity of the polarization component are not only strongly influenced by the void geometry, but also by the orientation of the void with reference to the detecting electrode $\left(\vec{\nabla} \lambda_{0}\right)$.

Similarly, the void geometry and its orientation have a significant influence on the critical permittivity ratios associated with the condition $q_{P n} / q_{n}=0$. The greater influ- 
ence is exhibited by the parallel orientation. However, with the exception of oblate voids in the parallel orientation, these permittivity ratios lie within the range 0.4 to $<1$. Hence the probability of this condition occurring in practice is high.

\subsection{PRACTICAL ASPECTS}

From equation (1) it is clear that the polarization component $q_{P}$ associated with the void geometry and orientation is directly reflected in the Poissonian induced charge $q$. Consequently measurement of $q$ on practical equipment with heterogeneous insulation will invariably contain an unknown, potentially harmless, but nevertheless confusing, polarization component. This component will, depending on its polarity, either add to or subtract from the component directly related to the void PD, $q_{\mu}$. Thus, in practice, lacking knowledge about void geometry and orientation, it will be impossible to quantify accurately from the recorded data the magnitude of the damaging void PD. Such a limitation leads directly to the conclusion that pattern recognition is the only viable approach to interpreting PD measurements. Evidence for this situation is reflected in the very many papers addressing the topic of pattern recognition which have appeared in the literature in recent years.

Reference to associated practical aspects may be found in [3].

\section{CONCLUSION}

$\mathrm{T}$ HE behaviour of the polarization component of the Poissonian induced charge with respect to void geometry and its orientation has been examined. For a heterogeneous bulk dielectric system, it is demonstrated that, in addition to the bulk dielectric permittivity ratio, the two void parameters have each a major influence on the magnitude and polarity of the polarization component. This affects directly the magnitude of the resultant Poissonian induced charge: i.e. the magnitude of the recorded partial discharge transient.

With reference to practical equipment, knowledge of void geometry and orientation will, in general, be non-existent. This situation implies that to diagnose correctly the magnitude of PD activity within the void, and thereafter to predict reliably component lifetimes presents a problematic exercise for heterogeneous bulk dielectric systems.

\section{REFERENCES}

[1] A. Pedersen, G. C. Crichton and I. W. McAllister, "The Theory and Measurement of Partial Discharge Transients", IEEE Trans. Electr. Insul., Vol. 26, pp. 487-497, 1991.

[2] A. Pedersen, G. C. Crichton and I. W. McAllister, "The Functional Relation Between Partial Discharges and Induced Charge", IEEE Trans. Dielectr. Electr. Insul., Vol. 2, pp. 535-543, 1995.

[3] I. W. McAllister and G. C. Crichton, "Influence of Bulk Dielectric Polarization upon Partial Discharge Transients: Effect of Heterogeneous Dielectric Geometry", IEEE Trans. Dielectr. Electr. Insul., Vol. 7, pp. 124-132, 2000.

[4] A. Pedersen, G. C. Crichton and I. W. McAllister, "Partial Discharge Detection: Theoretical and Practical Aspects", IEE Proc. - Sci., Measur. Techn., Vol. 142, pp. 29-36, 1995.

[5] G. C. Crichton, P. W. Karlsson and A. Pedersen, "Partial Discharges in Ellipsoidal and Spheroidal Voids", IEEE Trans. Electr. Insul., Vol. 24, pp. 335-342, 1989.

[6] I. W. McAllister, "Partial Discharges in Spheroidal Voids: Void Orientation", IEEE Trans. Dielectr. Electr. Insul., Vol. 4, pp. $456-461,1997$.

This paper is based on presentations given at the Eighth International Symposium on Gaseous Dielectrics, Virginia Beach, VA, 1998 and at the Conference on Electrical Insulation and Dielectric Phenomena, Austin, TX 1999.

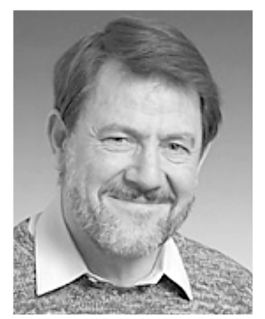

Iain W. McAllister (M'84-SM'91) was born in Glasgow, Scotland in 1942. He was educated at the University of Strathclyde, Glasgow, and received the B.Sc. and Ph.D. degrees from that University in 1964 and 1974, respectively. In 1972 he moved to Denmark to join Pedersen's group at the Technical University of Denmark, from which he retired in 2004. After a decade of research on long sparks in atmospheric air and corona onset phenomena, he began placing emphasis on the use of field theory in his research. This led to a greater diversity in the number of topics studied: e.g. surface roughness effects, charge accumulation in dc cables, partial discharge transients, electrostatic probe measurements, influence of surface conductivity. He has published over a 100 papers, and is an active referee for several international journals.

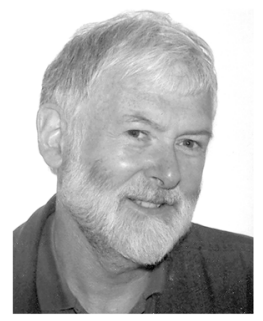

George C. Crichton (M'00) was born in Pewsey, England in 1940. He was educated at the University of Strathclyde, Glasgow, Scotland where he received the B.Sc. and $\mathrm{Ph}$. D. degrees in 1963 and 1969, respectively. He took up a lecturing appointment at the Technical University of Denmark in 1967. By his retirement in 2001 he had authored some 100 papers in the area of electrical insulation phenomena. He now pursues an active hobby as an amateur astronomer at the Dr. N. P. Wieth-Knudsen Observatory, Tisvilde, Denmark. 